\title{
ARTICLE
}

\section{Orbital mycoses in an adult subtropical population}

\author{
Allister S. Lee ${ }^{1,2} \cdot$ Princeton W. Y. Lee $^{1} \cdot$ Anthony Allworth $^{3} \cdot$ Tai Smith $^{4} \cdot$ Timothy J. Sullivan $^{1,2}$
}

Received: 7 April 2019 / Revised: 28 October 2019 / Accepted: 15 November 2019 / Published online: 10 December 2019

(c) The Author(s), under exclusive licence to The Royal College of Ophthalmologists 2019

\begin{abstract}
Background/objectives To report the spectrum of fungal infections involving the orbit encountered in an Australian subtropical population with respect to presentation, host risk factors, involved pathogens, treatment and outcomes.

Subjects/methods A retrospective chart review was performed on all adult patients with orbital mycosis treated by the senior author (TJS) from 1986 to 2017 in a tertiary setting.

Results Thirty cases of fungal infection involving the orbit were included in this case series. Of these, 26 patients had invasive disease and four patients had non-invasive disease. Causative organisms included mucormycosis (16), aspergillus (8) and other fungi (7). Common risk factors included haematological disorders or malignancy, neutropenia, corticosteroid use and diabetes mellitus. Mucormycosis in three immunocompetent patients was caused by Apophysomyces elegans. Orbital apex syndrome was observed in approximately one third of patients at initial ophthalmological assessment. Amphotericin B was used in most cases of mucormycosis, while there was a more varied spectrum of anti-fungal use in other fungal infections. Seven patients with mucormycosis proceeded to orbital exenteration with a survival rate of $43 \%$. No patients with other orbital fungal infections were exenterated.

Conclusions Orbital mycoses are not only opportunistic but true pathogenic infections. While initial symptoms may be varied, the development of orbital apex syndrome should raise suspicion for this condition, regardless of patient immune status or age. Survival and visual outcomes are often poor with invasive disease. Multidisciplinary team management with early orbital specialist involvement is essential.
\end{abstract}

This paper has been presented at the British Oculoplastics Surgery Society (BOPSS) annual meeting in Dublin, Ireland (13-15th June 2018) and European Society of Ophthalmic Plastic and Reconstructive Surgery (ESOPRS) annual meeting in Bucharest, Romania (13-15th September 2018).

Supplementary information The online version of this article (https:// doi.org/10.1038/s41433-019-0733-3) contains supplementary material, which is available to authorized users.

Allister S. Lee

allister.lee@uqconnect.edu.au

1 Department of Ophthalmology, Royal Brisbane and Women's Hospital (RBWH), Brisbane, QLD, Australia

2 University of Queensland, Brisbane, QLD, Australia

3 Department of Infectious Diseases, Royal Brisbane and Women's Hospital, Brisbane, QLD, Australia

4 Queensland Eye Institute, Brisbane, Australia

\section{Introduction}

Fungal infections involving the orbit are rare but often sight and life-threatening conditions. Mucormycosis is caused by fungi belonging to the order of Mucorales, which include Mucor, Rhizopus, Rhizomucor, Absidia, Apophysomyces, Cunninghamella and Saksenaea [1]. Aspergillosis has also been implicated as a significant cause of fungal infection involving the orbit [2]. In immunocompetent individuals, aspergillosis may cause non-invasive allergic fungal rhinosinusitis with orbital manifestations [3]. However, in immunosuppressed individuals, aspergillosis may develop into an acute, fulminant invasive disease which is more commonly seen with mucormycosis [2]. These infections are characterized by angioinvasion and tissue necrosis, with potentially serious consequences such as retinal, optic nerve, or orbital ischemia and further progression into the cranial cavity.

Risk factors for orbital mycoses have been well documented in previous studies. Haematological malignancy, solid-organ transplantation, diabetes mellitus, iron overload and deferoxamine therapy are among established risk 
factors for mucormycosis [1, 2]. Survival rates have been reported between 30 and $80 \%$ for mucormycosis; however, remains particularly low for those with leukaemia, lymphoma and solid-organ transplant [4-7].

Early diagnosis and aggressive treatment has been advocated by some authors in the management of invasive fungal disease [2, 8]. The routine administration of amphotericin B particularly in its liposomal form has been found to reduce mortality in mucormycosis [4, 5]. Some authors advocate aggressive surgical debridement with orbital exenteration for mucormycosis [9], whereas others have demonstrated survival with a more conservative approach without exenteration [10]. Nonetheless there remains a lack of consensus regarding the indications for exenteration, with the decision to perform this procedure resting primarily on the judgement of the treating physicians [11]. Similarly, the role of exenteration in other orbital fungal infections remains unclear.

In this case series, we aim to report the spectrum of orbital mycoses encountered in an Australian subtropical population with respect to presentation, involved pathogens, host risk factors, treatment and outcomes. We also propose our guidelines for the role of exenteration in sino-orbital fungal infections. We use the term "orbital mycoses", to incorporate all fungal infections involving the orbit including invasive and non-invasive forms.

\section{Methods}

We retrospectively reviewed the medical records of adult patients who were treated for fungal infections involving the orbit by the senior author in south-east Queensland, Australia between 1986 and 2017. All patients were referred to the senior author (TJS), who directs the largest public tertiary orbital service in our catchment area draining an estimated state population of over 5 million and local city population of 2.5 million [12]. Patients managed locally in regional centres or other institutions were not included. Patient management occurred in conjunction with otorhinolaryngology and infectious disease specialists. Two cases (patients 14 and 19) were previously published as case reports but have been included in this series for completeness [13, 14]. Institutional Review board (IRB) approval was obtained for this study. Consent was obtained from patients to publish clinical photographs (Figs. 1 and 2).

We collected data on age, sex, risk factors, presenting clinical features, causative pathogens, treatment and outcomes. Statistical significance between groups was calculated using Fisher's exact tests.

Infections were classified as "invasive" if meeting the criteria for proven or probable invasive fungal disease proposed by the European Organisation for Research and
Treatment of Cancer/Invasive Fungal Infections Cooperative Group and the National Institute of Allergy and Infectious Diseases Mycoses Study Group [15]. Proven invasive fungal infection was defined as histopathologic, cytopathologic or direct microscopic examination showing hyphae from biopsy specimen with evidence of associated tissue damage; or positive culture result for a sample obtained by sterile procedure from a normally sterile and clinically or radiologically abnormal site [15]. Probable invasive fungal infection was defined as the presence of combined host factors, clinical criterion and mycological criterion [15]. Infections without histopathological or radiological evidence of tissue invasion were classified as "non-invasive". We defined orbital involvement as the presence of ophthalmic clinical (e.g. proptosis) or imaging manifestations.

\section{Results}

\section{Patient and fungal characteristics}

We included 30 patients with orbital mycoses over approximately three decades (Supplementary Tables 1, 2). Age ranged from 23 to 82 years (mean 57 years) and 21 (70 $\%$ ) patients were male (Table 1). Common host risk factors and distribution of fungi are shown in Table 1 . There were 26 cases of invasive disease $(87 \%)$ and four cases of noninvasive disease (13\%). One patient was co-infected with Rhizopus spp. and Scedosporium prolificans (patient 11).

$13 / 26(50 \%)$ cases of invasive disease affected patients with haematological malignancy. Neutropenia was found in $7 / 13(54 \%)$ patients with haematological malignancy and in both patients with blood dyscrasia (myelodysplastic syndrome and aplastic anaemia). Five mucormycosis infections were in diabetic patients, including one who presented in diabetic ketoacidosis. All three mucormycosis infections in immunocompetent, non-diabetic patients, were caused by Apophysomyces elegans. One patient (patient 9) developed mucormycosis 3 weeks after renal transplant while receiving immunosuppressive therapy. All four patients with noninvasive disease (patients 21, 23, 25 and 29) were immunocompetent and most had a history of chronic sinusitis.

\section{Presenting clinical features}

The presenting symptoms and signs ranged from nasopharyngeal, facial to posterior orbital in nature (Table 2). Orbital apex syndrome (OAS) was observed in eight patients $(27 \%)$ at initial ophthalmic assessment. Of these, six had mucormycosis and four were immunocompetent. Two patients with aspergillosis developed OAS. Time duration from symptom onset to initial review by an 
Fig. 1 Case Example 1: Top left: Axial computed tomography slice through midorbits. Bottom left: Axial T2 magnetic resonance imaging scan. Top right: clinical presentation. Bottom right: clinical photograph of patient at 1-year follow-up. photograph of patient on

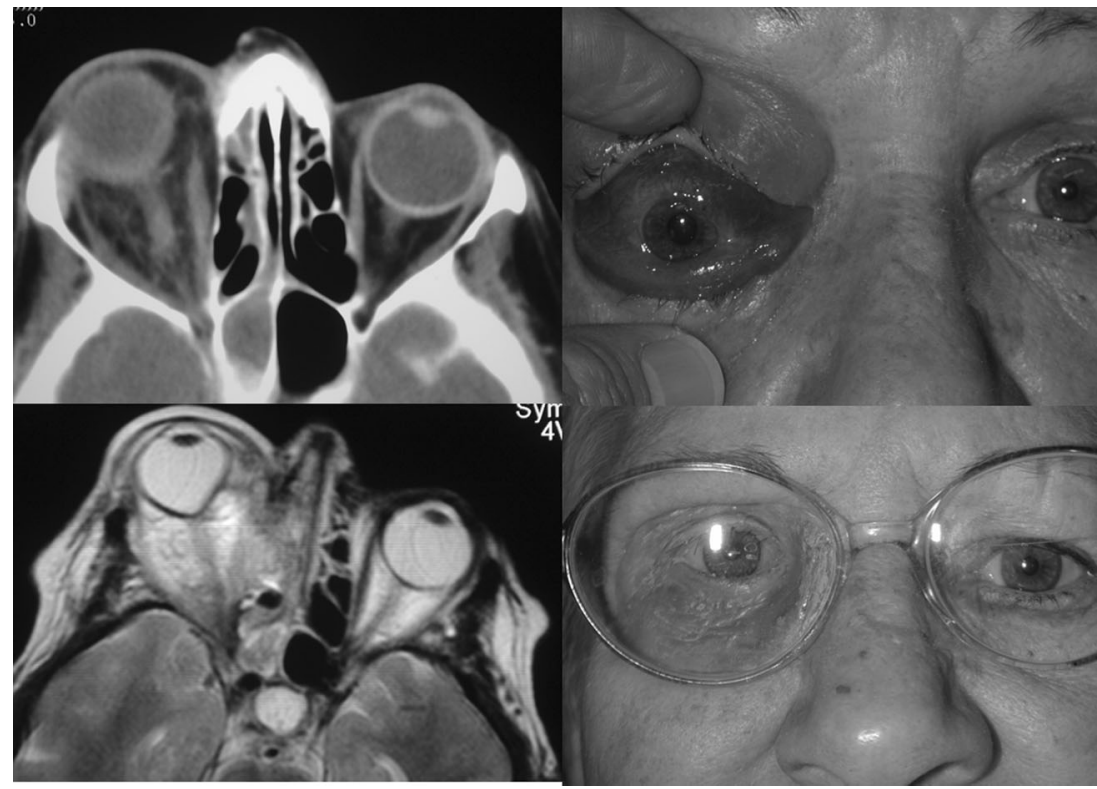

Fig. 2 Case Example 2: Top Left: Clinical photograph of patient on presentation. Top Right: Clinical photograph of patient at 6-month follow-up. Bottom: Axial T1 magnetic resonance imaging scan through the mid-orbits with gadolinium contrast.

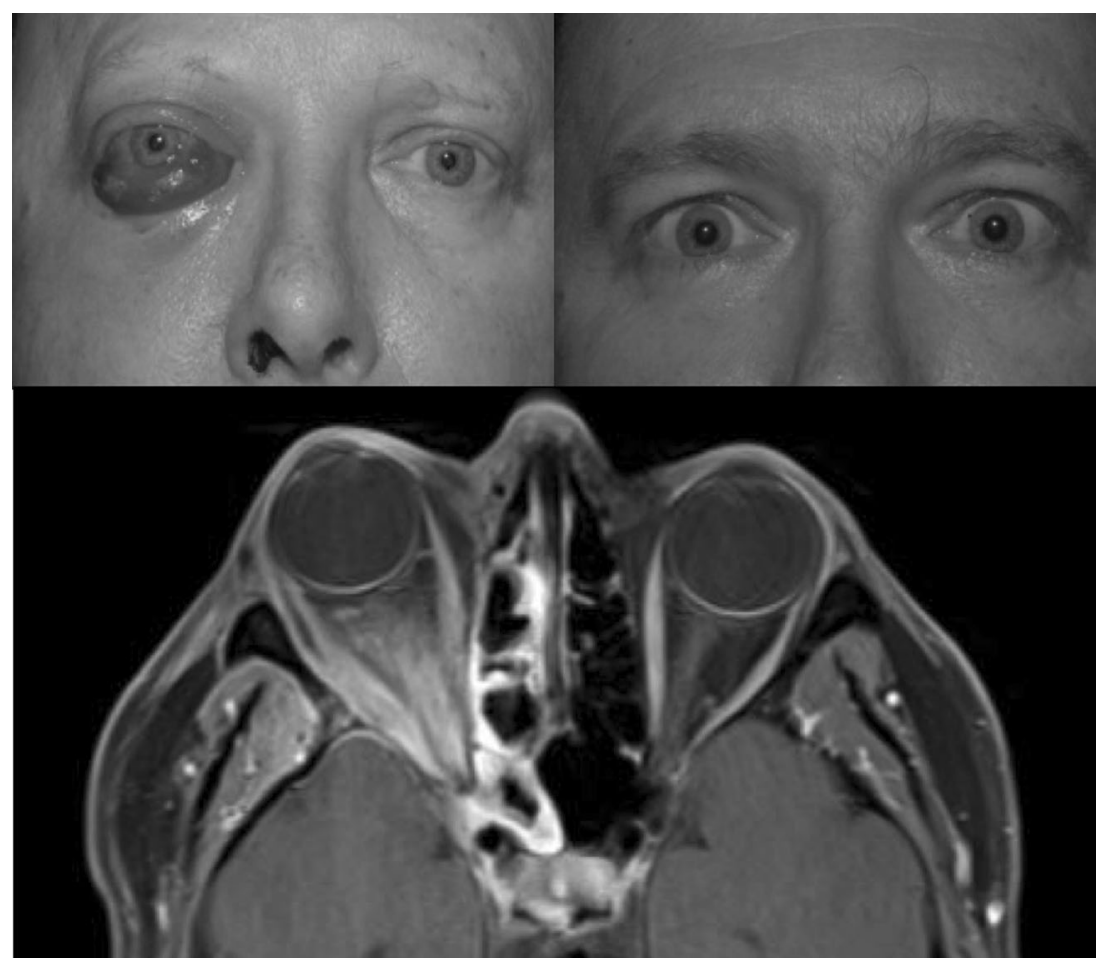

ophthalmologist was variable. Fifteen patients (50\%) were reviewed within 1 week of symptom onset, many of whom were already hospital inpatients for management of their underlying medical condition. Patients with mucormycosis had initial ophthalmology review on average earlier than patients with aspergillosis (mean $7 \mathrm{vs}$ 49 days). Similarly, patients with invasive disease were reviewed earlier than patients with non-invasive disease (mean 18 vs 28 days).

\section{Treatment and outcomes}

Conventional or liposomal amphotericin B (AMB) was used in most mucormycosis infections, while other fungal infections were treated with a more varied spectrum of anti-fungal agents (Table 3, Supplementary Table 2). Twenty-nine (97\%) patients underwent surgical biopsy with or without debridement. On average, patients with mucormycosis had more operations than patients infected with 
Table 1 Patient and fungal characteristics.

\begin{tabular}{lcc}
\hline Mean age (range) & & $57(23-82)$ \\
Male (\%)/female (\%) & $21(70) / 9(30)$ \\
Risk factors, $n$ (\%) & \\
$\quad$ Haematological malignancy/dyscrasia \\
$\quad$ Chemotherapy \\
$\quad$ Neutropenia \\
Sinusitis
\end{tabular}

${ }^{a}$ Including corticosteroid use

other fungi (Table 3). Seven patients with mucormycosis proceeded to exenteration with a survival rate of $43 \%$. Among the three exenterated patients who survived (patients 7, 14 and 16), two were infected with Apophysomyces elegans and only one was immunocompromised due to receiving corticosteroid and methotrexate therapy. No patients with other types of fungal infections were exenterated. 15/23 patients (65\%) who were not exenterated survived, although five had significantly impaired vision at follow-up including no perception of light (2), perception of light (1), counting fingers (1) and persistent diplopia (1). Survival rate differences when comparing exenterated and non-exenterated patients with mucormycosis or any invasive fungal infection were not statistically significant $(p=$ 1.00 and 0.67 , respectively). Overall survival rate was 59\% with nearly half of the surviving patients (including exenterated patients) having poor visual outcome. Survival rate for invasive aspergillosis (83\%) was almost double the survival rate for mucormycosis $(44 \%)$, however this was not statistically significant $(p=0.16)$.
Table 2 Presenting symptoms and signs.

\begin{tabular}{lc}
\hline Clinical feature & Number $(\%)$ \\
\hline Proptosis & $24(80)$ \\
Facial or eye pain & $22(73)$ \\
Ophthalmoplegia & $22(73)$ \\
Facial or periorbital swelling & $20(67)$ \\
Eyelid swelling & $15(50)$ \\
Rhinorrhea or sinusitis & $14(47)$ \\
Chemosis & $13(43)$ \\
Headache & $11(37)$ \\
Fever & $10(33)$ \\
RAPD & $10(33)$ \\
Visual changes & \\
Infraorbital hypoaesthesia & $10(33)$ \\
Ptosis & $7(23)$ \\
Altered sensorium & $7(23)$ \\
Retina ischemia & $5(17)$ \\
\hline
\end{tabular}

$R A P D$ relative afferent pupillary defect

${ }^{a}$ Include subjective vision changes, such as blurred vision and diplopia

Putative causes of death in most patients were the combination of extensive or disseminated fungal infection and progression of underlying life-threatening conditions. Two patients died due to infection with Scedosporium prolificans (patients 11 and 30) and one patient died from infection with Exserohilum species (patient 26). All patients with noninvasive disease survived and had good visual outcomes.

\section{Case example 1: Survival with orbital exenteration (patient 16)}

A 61year-old immunocompetent female presented with several days of worsening right-sided painful periorbital oedema and decreased vision. On examination, she was febrile with right-sided chemosis and proptosis (Fig. 1). Visual acuity OD was NPL with associated relative afferent pupillary defect (RAPD). There was complete external ophthalmoplegia and infraorbital hypoaesthesia. A diagnosis of orbital apex syndrome was made and intravenous antibiotics were initiated. Computed tomography showed opacification of the right ethmoid, sphenoid and frontal sinuses with orbital fat stranding. A sphenoid biopsy was performed, and tissue histopathology revealed broad non-septate hyphae with features of angioinvasion, consistent with mucormycosis. Magnetic resonance imaging post biopsy confirmed involvement of the posterior orbit (Fig. 1). Tissue culture later identified the pathogen as Apophysomyces Elegans. Liposomal amphotericin B was started, and the patient underwent three further debridements including orbital exenteration $\sim 2$ weeks after symptom onset. The patient recovered over the 
Table 3 Treatment and outcomes.

\begin{tabular}{|c|c|c|c|c|c|}
\hline & \multirow{2}{*}{$\begin{array}{l}\text { Mucormycosis }(n=16) \\
\text { I }(n=16)\end{array}$} & \multicolumn{2}{|c|}{ Aspergillus $(n=8)$} & \multicolumn{2}{|c|}{ Other fungi $(n=6)^{\mathrm{b}}$} \\
\hline & & $\mathrm{I}(n=6)$ & NI $(n=2)$ & $\mathrm{I}(n=4)$ & $\mathrm{NI}(n=2)$ \\
\hline \multicolumn{6}{|l|}{ Treatment, $n$} \\
\hline Amphotericin $\mathrm{B}^{\mathrm{a}}$ & $15 / 16$ & $4 / 6$ & $0 / 2$ & $3 / 4$ & $0 / 2$ \\
\hline Other anti-fungal agent & $7 / 16$ & $5 / 6$ & $0 / 2$ & $2 / 4$ & $1 / 2$ \\
\hline Orbital exenteration & $7 / 16$ & $0 / 6$ & $0 / 2$ & $0 / 4$ & $0 / 2$ \\
\hline $\begin{array}{l}\text { Sinus/orbital biopsy or } \\
\text { debridement, mean (range) }\end{array}$ & $2.5(0-8)$ & $1.5(1-2)$ & $1(1)$ & $1(1)$ & $2(2)$ \\
\hline \multicolumn{6}{|l|}{ Survival, $n(\%)$} \\
\hline All & 7/16 (44) & $5 / 6(83)$ & $2 / 2(100)$ & $2 / 4(50)$ & $2 / 2(100)$ \\
\hline Exenterated patients & $3 / 7(43)$ & - & - & - & - \\
\hline Poor visual outcome, $n(\%)^{\mathrm{c}}$ & $5 / 7(71)$ & $2 / 5(40)$ & $0 / 2(0)$ & $1 / 2(50)$ & $0 / 2(0)$ \\
\hline
\end{tabular}

$I$ invasive, $N I$ non-invasive

${ }^{a}$ Includes conventional, liposomal and lipid complex forms

${ }^{\mathrm{b}}$ Excluding patient co-infected with Rhizopus spp. and Scedosporium prolificans

${ }^{c}$ Poor visual outcome includes counting fingers, perception or no perception of light, no eye and persistent diploplia

next month and was subsequently discharged. She underwent successful prosthetic rehabilitation (Fig. 1) and remains infection free at 4-year follow-up.

\section{Case example 2: Survival without orbital exenteration (patient 10)}

A 51-year-old male receiving chemotherapy for T-cell lymphoblastic lymphoma was admitted to hospital for febrile neutropenia with a neutrophil count of $0.36 \times 10^{3} / \mathrm{mcL}$. The patient developed right-sided facial pain, swelling and paraesthesia with blurred vision. Magnetic resonance imaging revealed T2 hypointense, non-enhancing tissue of the right maxillary sinus with extension into the inferior orbit and pterygopalatine fossa. On examination there was proptosis, painful restricted ocular motility in all directions, marked chemosis and conjunctival injection (Fig. 2). There were no signs of optic neuropathy or retinal ischemia. Liposomal amphotericin B was initiated and the patient proceeded to have endoscopic sinus debridement by the otolaryngologists. Histopathology revealed devitalised tissue with haemorrhagic infarction and Periodic Acid-Schiff (PAS) positive fungal hyphae suggestive of mucormycosis. Oral posaconazole was prescribed and the fungus was later identified as Rhizomисоr miehei on culture. Over 2 weeks the patient's visual acuity deteriorated to 6/20 OD with Grade 2 RAPD and optic disc pallor. Repeat MRI showed extension of disease, involving the temporal lobe (Fig. 2). His posacaonzole dose was increased and a collective multidisciplinary decision was made to not perform further surgery. However, with maximal medical therapy, the patient gradually improved and was eventually discharged $\sim 6$ weeks after symptom onset. At 6-month follow-up his optic neuropathy had completely resolved with VA OD 6/7.5; however, he had persistent upgaze palsy with associated diplopia. The patient continued posaconazole for 18 months total, with no evidence of disease recurrence at 4-year follow-up.

\section{Discussion}

In this case series of orbital mycoses, we identified 16 cases of mucormycosis, eight cases of aspergillosis and seven cases of infection due to other fungi. We believe this would encompass a significant proportion of cases of fungal infection involving the orbit over the past 31 years in our catchment, excluding patients who were palliated immediately due to fulminant, disseminated disease and required no orbital specialist input, or were managed locally in regional centres or other facilities. The majority of patients (87\%) suffered from invasive disease. Approximately half were immunocompromised, many of whom were neutropenic. Presenting symptoms and signs were variable; however, orbital apex syndrome was found in nearly one third of cases. Seven mucormycosis patients underwent exenteration with three surviving (43\%). Nine were managed without exenteration with four surviving (44\%). Although there was no significant difference in survival, exenteration was an important component of management in patients with extensive amounts of infarcted, necrotic, or dead orbital tissue. No patients with other fungal infections were exenterated. Favourable prognostic factors for survival among the exenterated group include infection by Apophysomyces elegans and the absence of neutropenia or haematological malignancy. Even in surviving patients of invasive disease, visual outcome was usually poor. 
Our results showed that orbital mycoses could affect any individual regardless of their underlying immune status and age. Hence, they should be considered a true pathogenic condition rather than an opportunistic infection. The course of the disease is influenced by the interaction between the host immune system and the virulence of the causative fungi. Our study suggests that orbital infection due to Apophysomyces elegans seems to carry a better prognosis compared to other fungi. This is consistent with data from previous studies, which reported favourable survival outcomes in patients with Apophysomyces elegans [14, 16, 17]. The more favourable prognosis may be in part explained by the functioning immune system typically seen in these cases. Nonetheless, infection from this fungus has been associated with serious sequelae including orbital ischaemia, cavernous sinus thrombosis and osteomyelitis [18]. In contrast, infections due to Scedosporium and Cunninghamella have poor survival outcome and this may be explained by their resistance to amphotericin [1, 19]. Exserohilum spp. have been reported to cause invasive infection in both immunocompromised and immunocompetent hosts [20]. This is supported by our study, as one of our patients infected with Exserohilum had acute leukaemia and the other was immunocompetent.

Our results enforce that neutropenia is a risk factor for invasive orbital mycoses, particularly in patients with haematological malignancy or solid-organ transplant. Neutrophils are thought to play a major role in overcoming fungal infection and their recovery has been shown to be a significant factor for recovery from mucormycosis in haematologic malignancy [1, 4]. Interestingly, our single patient who developed mucormycosis in the context of immunosuppression following renal transplant survived. Immunosuppressive agents used to prevent graft rejection also weaken the immune response to fungi, which explains the poor survival rate among transplant recipients [2]. However, any reduction in the immunosuppressive regime should always be coordinated by the treating physicians as the risk of graft rejection must be carefully balanced against the potential improvement in recovery from infection.

Previous studies show that Human Immunodeficiency Virus (HIV) does not appear to increase the risk of developing mucormycosis, except in the context of intravenous drug use [1]. This is probably because neutrophils, not T-lymphocytes, are involved in the protection against mucormycosis. Similarly, invasive aspergillosis is more commonly seen in HIV patients with drug-induced neutropenia [21]. Our single patient with HIV developed orbital aspergillosis in the context of chronic liver disease due to hepatitis $\mathrm{C}$ and alcohol abuse with no history of intravenous drug use or neutropenia [13]. However, Pellicelli et al. suggest that neutrophil phagocytosing potential is lower in patients with liver cirrhosis, leading to increased susceptibility to fungal infections [22].
The relatively high prevalence of orbital apex syndrome in our study should prompt clinicians to strongly consider orbital mycoses early in the differential diagnosis when encountering this presentation, which may have infectious, inflammatory, neoplastic or traumatic causes [23]. Prompt diagnosis and treatment have been cited as good prognostic factors in mucormycosis, and therefore timely recognition of these infections is important [9]. Our spectrum of presenting symptoms and signs are similar with those reported in other literature $[9,22]$. In our study, proptosis, ophthalmoplegia and periorbital pain or swelling were the most common orbital signs in cases of both invasive and non-invasive disease.

Amphotericin B has been associated with improved recovery from mucormycosis, particularly in its liposomal form $[1,2,4,5,8]$. Other measures such as daily irrigation and packing of the involved orbit and sinuses with $\mathrm{AMB}$ have been reported to increase drug delivery directly to the infected tissues with improved chances of recovery from mucormycosis [2, 24]. Hirabayashi et al. recently reported a novel case of successfully treated orbital mucormycosis with retrobulbar injection of $\mathrm{AMB}$ without exenteration [25]. Intraorbital injection of AMB has also been shown to be effective palliative therapy in patients unable to undergo aggressive surgical debridement [26]. Overall, there have been six reported cases of retrobulbar injection of AMB for orbital fungal infection with generally favourable outcomes [27]. In patients with non-zygomatic fungal infections, there is evidence supporting the use of other systemic anti-fungal agents. For example, a randomized clinical trial has shown superior outcomes with voriconazole compared to AMB in treating patients with invasive aspergillosis [28]. None of our patients received hyperbaric oxygen therapy; however, there is some evidence showing that it may increase survival rate in patients through multiple mechanisms such as optimising fungicidal ability of neutrophils [29].

Several authors have advocated for aggressive debridement of necrotic tissue in invasive disease to decrease the total fungal burden $[4,9]$. In our study, seven patients with mucormycosis underwent orbital exenteration and less than half survived. Of exenterated patients who survived, most were immunocompetent except one patient who was receiving corticosteroids and methotrexate for rheumatoid arthritis. Therefore, it is difficult to attribute their survival to having this intervention. There is a lack of guideline on the indications for exenteration in orbital mycoses. In a review of 292 cases of orbital mucormycosis, Hargrove et al. found that orbital exenteration was associated with increased survival only in patients presenting with fever [13]. Our rationale for performing exenteration was to remove the bolus of ischemic or necrotic tissue which allows the fungus to propagate into adjacent non-ischemic tissues, resulting in further spread of the disease (e.g. patient 16, Fig. 1). We did not perform exenteration in patients in whom we believed 
we could achieve cure with medical treatment and less invasive surgery (e.g. patient 10, Fig. 2). In other fungal infections such as orbital aspergillosis, some authors justify exenteration in cases with significant intraconal or orbital apex involvement [30, 31]. Interestingly, most of our patients with aspergillosis who demonstrated posterior orbital signs (including one with OAS) survived without exenteration. This could support a less radical approach in the surgical management of orbital aspergillosis, compared to mucormycosis.

All patients with non-invasive disease in this study had allergic fungal rhinosinusitis (AFS) with orbital involvement. Causative fungi are consistent with those cited in literature, including Aspergillus, Bipolaris and Curvularia [32]. AFS typically affects immunocompetent individuals from warm, humid climates and is an endemic in locations such as the Middle East [32]. The disease arises from a host hypersensitivity reaction to fungi residing in the sino-nasal tract [32]. Although it initially presents as chronic rhinosinusitis, expansion of involved sinus cavities may lead to ophthalmic manifestations such as proptosis, ophthalmoplegia and in rare cases, compressive optic neuropathy with vision loss [33]. Treatment usually includes endoscopic sinus debridement, to allow drainage of the thick allergic mucin that is characteristically found in this disease $[4,32]$. In contrast to invasive fungal infection, AFS is typically treated with systemic corticosteroids rather than anti-fungal medication [34]. Outcomes are generally favourable, with all our patients surviving and having normal vision at follow-up.

Our study is limited by the small sample size, particularly regarding cases of non-invasive disease and the retrospective study design. However, orbital mycoses are rare infections and it would be difficult to conduct a large, prospective study with sufficient power. Nonetheless, we have presented the largest case series of orbital mycoses in a subtropical population to date. In addition, we believe our case mix may represent more complex and advanced disease as all patients were referred to and managed by our tertiary orbital service. Patients with less severe disease could have been appropriately managed at smaller institutions and would not be included in our study. Therefore, our reported experience with orbital mycoses may not necessarily reflect those in another setting where not only climate factors but also population factors may be different. Another limitation is that we do not have complete data on the use of granulocyte infusions for our neutropenic patients. A few studies have reported using granulocyte or granulocyte-macrophage colony-stimulating factors as an adjunctive treatment for mucormycosis in neutropenic patients [35, 36].

Orbital mycoses are potentially life-threatening conditions, which affect both immunosuppressed and immunocompetent individuals of any age. In this case series, we found that the presenting clinical features were variable, and clinicians should always consider this diagnosis in at risk individuals. Development of the orbital apex syndrome should prompt clinicians to consider invasive fungal infection regardless of their immune status. Compromised immunity due to underlying non-reversible disease is a poor prognostic factor in affected patients. Multidisciplinary management with oculoplastics, otorhinolaryngologists, haematologists, transplant, infectious disease physicians and intensivists is essential. We believe the role of exenteration in the management of orbital mycoses, particularly mucormycosis, is to debride and remove extensively involved necrotic, non-viable orbital tissue, acknowledging that it has not been shown to improve survival in this series. Where there is minimal orbital involvement, as in the cases described by Athavale et al., [10] we agree that nonexenteration management is appropriate.

\section{Summary}

\section{What was known before}

- Presenting clinical features Presenting symptoms and signs vary from nasopharyngeal, facial to posterior orbital in nature.

- Management and outcomes Prompt anti-fungal therapy and surgical debridement improves survival, although the role of orbital exenteration remains unclear.

- Mortality rates are high, particularly in immunosuppressed patients with mucormycosis.

\section{What this study adds}

- Presenting clinical features Orbital apex syndrome is common on presentation (approximately one third of patients).

- Distribution of fungi A different spectrum of fungi may be observed from a subtropical population.

- Management and outcomes Survival appears favourable for Apophysomyces Elegans.

- Although not shown to improve survival in our study, orbital exenteration should be considered in select cases.

\section{Compliance with ethical standards}

Conflict of interest The authors declare that they have no conflict of interest.

Publisher's note Springer Nature remains neutral with regard to jurisdictional claims in published maps and institutional affiliations. 


\section{References}

1. Prabhu RM, Patel R. Mucormycosis and entomophthoramycosis: a review of the clinical manifestations, diagnosis and treatment. Clin Microbiol Infect. 2004;10:31-47.

2. Thomas PA. Current perspectives on ophthalmic mycoses. Clin Microbiol Rev. 2003;16:730-97.

3. Klapper SR, Lee AG, Patrinely JR, Stewart M, Alford EL. Orbital involvement in allergic fungal sinusitis. Ophthalmology. 1997; 104:2094-100.

4. Pagano L, Offidani M, Fianchi L, Nosari A, Candoni A, Piccardi $\mathrm{M}$, et al. Mucormycosis in hematologic patients. Haematologica. 2004;89:207-14.

5. Kontoyiannis DP, Wessel VC, Bodey GP, Rolston KV. Zygomycosis in the 1990s in a tertiary-care cancer center. Clin Infect Dis. 2000;30:851-6.

6. Ferry AP, Abedi S. Diagnosis and management of rhinoorbitocerebral mucormycosis (phycomycosis). A report of 16 personally observed cases. Ophthalmology. 1983;90:1096-104.

7. Husain S, Alexander BD, Munoz P, Avery RK, Houston S, Pruett $\mathrm{T}$, et al. Opportunistic mycelial fungal infections in organ transplant recipients: emerging importance of non-Aspergillus mycelial fungi. Clin Infect Dis. 2003;37:221-9.

8. Chakrabarti AD, Sharma A, Panda A, Das N, Gupta N, Sakhuja $\mathrm{KL}$, et al. Ten years' experience in zygomycosis at a tertiary care centre in India. J Infect. 2001;42:261-6.

9. Yohai RA, Bullock JD, Aziz AA, Markert RJ. Survival factors in rhino-orbital-cerebral mucormycosis. Surv Ophthalmol. 1994; 39:3-22.

10. Athavale DD, Jones R, O'Donnell BA, Forer M, Biggs N. Nonexenteration management of sino-orbital fungal disease. Ophthal Plast Reconstr Surg. 2017;33:426-9.

11. Hargrove RN, Wesley RE, Klippenstein KA, Fleming JC, Haik BG. Indications for orbital exenteration in mucormycosis. Ophthal Plast Reconstr Surg. 2006;22:286-91.

12. Australian Bureau of Statistics. Regional Population Growth, Australia, 2017-2018 [Internet]. Canberra: Commonwealth of Australia; 2019 [cited 2019 Aug 12]. ABS Catalogue No. 3218.0. http://abs.gov.au/AUSSTATS/abs@nsf/mf/3218.0.

13. Lee LR, Sullivan TJ. Aspergillus sphenoid sinusitis-induced orbital apex syndrome in HIV infection. Aust N. Z J Ophthalmol. 1995;23:327-31.

14. Fairley C, Sullivan TJ, Bartley P, Allworth T, Lewandowski R. Survival after rhino-orbital-cerebral mucormycosis in an immunocompetent patient. Ophthalmology. 2000;107:555-8.

15. De Pauw B, Walsh TJ, Donnelly JP, Stevens DA, Edwards JE, Calandra T, et al. Revised Definitions of Invasive Fungal Disease from the European Organization for Research and Treatment of Cancer/Invasive Fungal Infections Cooperative Group and the National Institute of Allergy and Infectious Diseases Mycoses Study Group (EORTC/MSG) Consensus Group. Clin Infect Dis. 2008;46:1831-1821.

16. Liang KP, Tleyjeh IM, Wilson WR, Roberts GD, Temesgen Z. Rhino-orbitocerebral mucormycosis caused by Apophysomyces elegans. J Clin Microbiol. 2006;44:892-8.

17. Sridhara SR, Paragache G, Panda NK, Chakrabarti A. Mucormycosis in immunocompetent individuals: an increasing trend. J Otolaryngol. 2005;34:402-6.
18. Wolkow N, Jakobiec FA, Stagner AM, Cunnane ME, Piantadosi AL, Basgoz N, et al. Chronic orbital and calvarial fungal infection with Apophysomyces variabilis in an immunocompetent patient. Surv Ophthalmol. 2017;62:70-82.

19. Ellis D. Amphotericin B: spectrum and resistance. J Antimicrob Chemother. 2002;49:7-10.

20. Gupta A, Xess I, Sharma SC, Mallik S Invasive rhinosinusitis by Exserohilum rostratum in an immunocompetent child. BMJ Case Rep. 2014; https://doi.org/10.1136/bcr-2013-202380.

21. Dagenais TR, Keller NP. Pathogenesis of Aspergillus fumigatus in Invasive Aspergillosis. Clin Microbiol Rev. 2009;22:447-65.

22. Pellicelli AM, D'Ambrosio C, Villani R, Cerasari G, Iaalongo P, Cortese A, et al. Liver Cirrhosis and Rhino-Orbital Mucormycosis, a Possible but Rare Association: description of a Clinical Case and Literature Review. Braz J Infect Dis. 2009;13:314-6.

23. Jiang $\mathrm{N}$, Zhao $\mathrm{G}$, Yang $\mathrm{S}$, Lin J, Hu L, Che C, et al. A retrospective analysis of eleven cases of invasive rhino-orbito-cerebral mucormycosis presented with orbital apex syndrome initially. BMC Ophthalmol. 2016;16:10.

24. Joos ZP, Patel BC. Intraorbital irrigation of Amphotericin B in the treatment of rhino-orbital mucormycosis. Opthal Plast Reconstr Surg. 2017;33:13-16.

25. Hirabayashi KE, Kalin-Hajdu E, Brodie FL, Kersten RC, Russell MS, Vagefi MR. Retrobulbar injection of amphotericin B for orbital mucormycosis. Ophthal Plast Reconstr Surg. 2017;33:94-97.

26. Cahill KV, Hogan CD, Koletar SL, Gersman M. Intraorbital injection of Amphotericin B for palliative treatment of Aspergillus orbital abscess. Ophthal Plast Reconstr Surg. 1994;10:276-77.

27. Kalin-Hajdu E, Hirabayashi KE, Vagefi MR, Kersten RC. Invasive fungal sinusitis: treatment of the orbit. Curr Opin Ophthalmol. 2017;28:522-33.

28. Herbrecht R, Denning DW, Patteron TF, Bennett JE, Greene RE, Oestmann JW, et al. Voriconazole versus amphotericin B for primary therapy of invasive aspergillosis. N. Engl J Med. 2002;347:408-15.

29. Wali U, Balkhair A, Al-Mujaini A. Cerebro-rhino orbital mucormyosis: an update. J Infect Public Health. 2012;5:116-26.

30. Adler SC, Isaacson G, Sasaki CT. Invasive aspergillosis of the paranasal sinuses and orbit: can you save the eye? Am J Otolaryngol. 1997;18:230-4.

31. Dhiwaker M, Thakar A, Bahadur S. Invasive sino-orbital aspergillosis: surgical decisions and dilemmas. J Laryngol Otol. 2003;117:280-5.

32. Montone KT. Pathology of fungal rhinosinusitis: a review. Head Neck Pathol. 2016;10:40-46.

33. Alaraj AM, Al-Faky YH, Alsuhaibani AH. Ophthalmic Manifestations of Allergic Fungal Sinusitis. Ophthalmic Plast Reconstr Surg. 2018;34:463-6.

34. Chang WJ, Tse DT, Bressler KL, Casiano RR, Rosa RH, Johnson TE. Diagnosis and management of allergic fungal sinusitis with orbital involvement. Ophthal Plast Reconstr Surg. 2000;16:72-4.

35. Garcia-Diaz JB, Palau L, Pankey GA. Resolution of rhinocerebral zygomycosis associated with adjuvant administration of granulocyte-macrophage colony-stimulating factor. Clin Infect Dis. 2001;32:e145-150.

36. Sahin B, Paydas S, Cosar E, Bicakci K, Hazar B. Role of granulocyte colony-stimulating factor in the treatment of mucormycosis. Eur J Clin Microbiol Infect Dis. 1996;15:866-9. 